$\mathrm{DOE} / \mathrm{BC} / 15211-4$

Distribution Category UC-122

A Note on the Evaporation of a Stagnant Liquid

\author{
By \\ Ioannis N. Tsimpanogiannis \\ Yanis C. Yortsos \\ A.K. Stubos
}

March 2000

Work Performed Under Contract No DE-AC26-99BC15211

Prepared for

U.S. Department of Energy

Assistant Secretary for Fossil Energy

Thomas B. Reid, Project Manager

National Petroleum Technology Office

P.O. Box 3628

Tulsa, OK 74101

Prepared by

Department of Chemical Engineering

University of Southern California

Los Angeles, CA 90089-1211

In collaboration with

National Center for Scientific Research "Demokritos"

15310 Aghia Paraskevi

GREECE 


\section{DISCLAIMER}

This report was prepared as an account of work sponsored by an agency of the United States Government. Neither the United States Government nor any agency thereof, nor any of their employees, make any warranty, express or implied, or assumes any legal liability or responsibility for the accuracy, completeness, or usefulness of any information, apparatus, product, or process disclosed, or represents that its use would not infringe privately owned rights. Reference herein to any specific commercial product, process, or service by trade name, trademark, manufacturer, or otherwise does not necessarily constitute or imply its endorsement, recommendation, or favoring by the United States Government or any agency thereof. The views and opinions of authors expressed herein do not necessarily state or reflect those of the United States Government or any agency thereof. 


\section{DISCLAIMER}

Portions of this document may be illegible in electronic image products. Images are produced from the best available original document. 


\section{Table of Contents}
ABSTRACT
NTRODUCTION

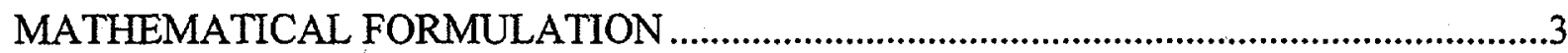

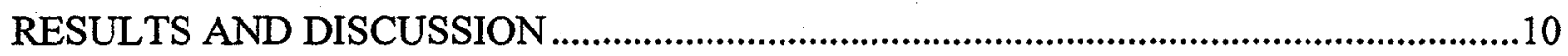

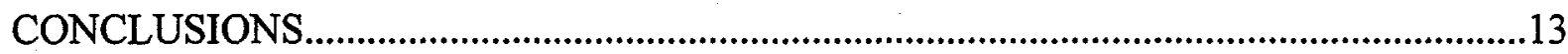

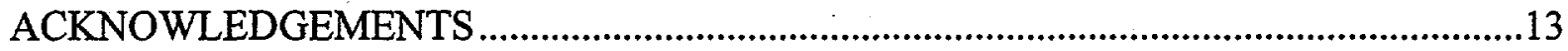

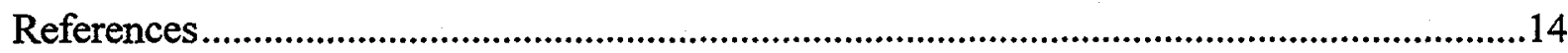

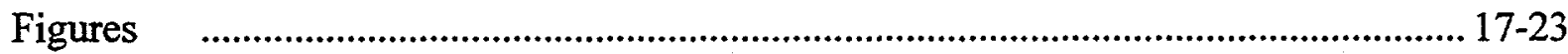




\title{
A NOTE ON THE EVAPORATION OF A STAGNANT LIQUID
}

\author{
Ioannis N. Tsimpanogiannis and Yanis C. Yortsos ${ }^{1}$ \\ Department of Chemical Engineering \\ University of Southern California \\ Los Angeles, CA 90089-1211 \\ and \\ Athanasios K. Stubos \\ National Center for Scientific Research "Demokritos" \\ 15310 Ag. Paraskevi, Greece \\ Submitted to Ind. Eng. Chem. Res. Journal, March 30, 1999. \\ Revised version submitted February 23, 2000.
}

\begin{abstract}
We model the evaporation of a stagnant liquid from an initially filled block to a flowing gas stream. The motivation for this problem arises from applications in drying of porous media, when the pressure is low, and in the recovery of oil from fractured reservoirs by gas injection, when the pressure is high. A similarity solution is developed that accounts for diffusion in both phases. Diffusion in the liquid phase can be important in high pressure applications, where the gas may dissolve in the liquid phase. The motion of the interface and the evaporation rates are calculated as a function of the various thermodynamic parameters for systems of interest, including $n$-alkanes and methane or nitrogen. The effect of counter-diffusion is shown to slow down the evaporation process, although not by an order of magnitude, in typical cases.
\end{abstract}

\footnotetext{
${ }^{1}$ Author to whom correspondence should be addressed. Tel.: (213) 740-0317. Fax: (213) 740-8053. E-mail: yortsos@archie.usc.edu
} 


\section{INTRODUCTION}

Of interest to certain reservoir engineering applications is the recovery of trapped or immobile oil by its evaporation and subsequent diffusion in a flowing gas. The typical application involves gas injection at high pressures in fractured reservoirs containing light oil (Le Gallo et al. [1]). Oil trapped inside matrix blocks of low permeability evaporates and diffuses in a gas injected at high pressures, which is flowing in the high-permeability fracture network surrounding the porous matrix (see schematic of Figure 1a). A similar problem, although at low pressures, arises in the remediation of contaminated soils, where trapped and immobile organic contaminants vaporize, diffuse and are convected away in a flowing gas (soil vapor extraction, Ho et al. [2]).

The problem involves a variety of processes: diffusion and convection in the gas phase, diffusion, capillary and viscous effects in the liquid phase, heat transfer and vapor-liquid equilibria. In a recent paper (Tsimpanogiannis et al. [3]) we analyzed in detail the motion of the liquid-gas interface in a porous medium, for the geometry described above, but under low pressure conditions and in the context of drying. In that work, emphasis was placed on the effect of capillarity and the pore structure on the scaling of the thickness of the drying front (Shaw [4]), using a thermodynamic description based on the dilute limit approximation. Ignored were effects of dissolution of the injected gas in the liquid and of liquid-phase diffusion, both of which are expected at higher pressures.

In this paper, we consider a version of the more general problem, with main objective the understanding of the effect of higher pressures on the velocity of the gas-liquid interface. Experimental evidence (Le Romancer et al. [5]) suggests that high-pressure gas injection results under certain conditions in a decrease of the rate of the interface motion, a result that may be due to gas dissolution and diffusion in the liquid phase. To explore this possibility, we consider in this paper a simple 1-D model of this process as illustrated schematically in Figure $1 \mathrm{~b}$. A liquid A, originally occupying a semi-infinite medium, is subject to evaporation and diffusion in a flowing gas $\mathrm{B}$, the composition of which at the top of the medium (at $z=0$ ) remains constant. Due to the possible high pressure in the gas, component B may solubilize and counter-diffuse in the liquid phase. For the sake of generality, the liquid is 
taken to consist initially of a binary mixture of $\mathrm{A}$ and $\mathrm{B}$, of mole fraction $x_{A \infty}$. Contrary to our previous work [3], we neglect effects of the pore microstructure, which give rise to 3-D fronts. Such effects can be included in a relatively straight forward analysis, but will not be considered here.

The isothermal, one-dimensional evaporation and diffusion of a solute through a stagnant gas in a tube or a channel, is often referred to as Stefan diffusion. The problem analyzed here can also be considered as a variation of the Stefan problem, under somewhat different process and boundary conditions. We note that in the standard references (e.g. Bird et al. [6], Slattery [7], and Taylor and Krishna [8]) unsteady-state evaporation is treated in the dilute-limit approximation, under the assumption that the gas-liquid interface is stationary. Slattery and Mhetar [9] considered evaporation of a liquid in a long vertical tube by relaxing the assumption of a stationary interface, while Mhetar and Slattery [10] modeled the problem of the evaporation of a binary liquid in the same geometry by also including diffusion in the liquid. Our problem is similar to the latter, although differences exist in the specific geometries considered, as well as in the fact that we consider solubilization and diffusion of component $\mathrm{B}$ in the liquid, which is expected at higher pressures.

In the following, we present the mathematical formulation of the problem, in which the porous medium is treated as an equivalent continuum. Because the emphasis is on understanding thermodynamic and transport effects, the formulation is relevant to the problem in a tube. It is certainly acknowledged that pore structure will affect diffusion, the formulation of the problem, and the motion and dimensionality of the interface. However, these effects are not in the scope of this investigation. A similarity solution is derived, based on which we analyze the effects of the ratio of diffusivities, pressure, initial composition and temperature on the velocity of the gas-liquid interface. The analysis provides insight on the relevant mass transfer and phase equilibria mechanisms, and can be used to model the more complex interface dynamics in an actual porous medium, where pore-structure effects are important.

\section{MATHEMATICAL FORMULATION}

Consider the problem described schematically in Figure 1b. The condensible component $A$ in the gas phase satisfies the mass balance 


$$
\frac{\partial c_{A}^{v}}{\partial t}+\frac{\partial N_{A z}^{v}}{\partial z}=0
$$

where $N_{A z}^{v}$ is the $z$-component of the molar flux of A in the gas phase, expressed for a binary mixture as

$$
N_{A z}^{v}=-c^{v} \mathcal{D}_{A B}^{v} \frac{\partial y_{A}}{\partial z}+y_{A}\left(N_{A z}^{v}+N_{B z}^{v}\right)
$$

Here, $c_{A}^{v}$ is the molar concentration, $y_{A}$ is the molar fraction of $\mathrm{A}\left(c_{A}^{v}=c^{v} y_{A}\right)$ and $\mathcal{D}_{A B}^{v}$ is the binary diffusion coefficient in the gas phase. Species $B$ is also conserved, hence

$$
\frac{\partial c_{B}^{v}}{\partial t}+\frac{\partial N_{B z}^{v}}{\partial z}=0
$$

By adding (1) and (3) and assuming that $c^{v}=c_{A}^{v}+c_{B}^{v}$ is constant at a fixed pressure, we get

$$
N_{A z}^{v}+N_{B z}^{v}=N_{z}^{v}(t)
$$

The assumption of spatially constant $c^{v}$ is quite reasonable for the systems and conditions considered below. Similar balances can be written for the liquid phase

$$
\frac{\partial c_{A}^{l}}{\partial t}+\frac{\partial N_{A z}^{l}}{\partial z}=0
$$

and

$$
N_{A z}^{l}=-c^{l} \mathcal{D}_{A B}^{l} \frac{\partial x_{A}}{\partial z}+x_{A}\left(N_{A z}^{l}+N_{B z}^{l}\right)
$$

where $c_{A}^{l}$ is the molar concentration and $x_{A}$ the molar fraction of $\mathrm{A}$ in the liquid phase, $\left(c_{A}^{l}=c^{l} x_{A}\right.$ ) and $\mathcal{D}_{A B}^{l}$ is the binary diffusion coefficient in the liquid phase. If, as in the gas phase, we were to also assume that the overall liquid molar concentration is constant, then

$$
N_{A z}^{l}+N_{B z}^{l}=N_{z}^{l}(t) \approx 0
$$

where the latter equation reflects the boundary condition of no-flux in the far-field. However, this assumption is not valid, in general, especially at higher pressures or when B is highly soluble. For the sake of simplicity, we will proceed first with the assumption of a spatially 
soluble. For the sake of simplicity, we will proceed first with the assumption of a spatially uniform liquid molar concentration, as this approximation allows for an exact solution to be obtained. The more general problem will be analyzed in more detail in a subsequent section. It will be shown that the error induced does not affect significantly the major conclusions of the paper. Implicit in the analysis to follow is also the assumption of spatially uniform diffusion coefficients. While the similarity solution to be developed does not rely on this assumption, we decided to adopt it for simplicity in the calculations and because its effect was found not to be significant.

\section{(i). Spatially constant overall molar concentration}

With the assumption of constant overall concentration, the balances for components $\mathrm{A}$ and $B$, equations (1) and (5), respectively, become

$$
\frac{\partial y_{A}}{\partial t}+\frac{N_{z}^{v}(t)}{c^{v}} \frac{\partial y_{A}}{\partial z}=\mathcal{D}_{A B}^{v} \frac{\partial^{2} y_{A}}{\partial z^{2}}
$$

and

$$
\frac{\partial x_{A}}{\partial t}=\mathcal{D}_{A B}^{l} \frac{\partial^{2} x_{A}}{\partial z^{2}}
$$

Concentration fields are coupled at the interface (subscript $e$ ) by the following mass balances for the two species $A$ and $B$

$$
\begin{aligned}
& M_{A} N_{A z}^{v}-\rho_{A e}^{v} v_{z}^{I}=M_{A} N_{A z}^{l}-\rho_{A e}^{l} v_{z}^{I} \text { at } z=f(t) \\
& M_{B} N_{B z}^{v}-\rho_{B e}^{v} v_{z}^{I}=M_{B} N_{B z}^{l}-\rho_{B e}^{l} v_{z}^{I} \text { at } z=f(t)
\end{aligned}
$$

where $\rho_{A e}^{l}$ and $\rho_{B e}^{l}$ denote mass densities of $A$ and $B$ in the liquid phase, respectively, hence $\rho_{A e}^{l}=x_{A e} c^{l} M_{A}$ and $\rho_{B e}^{l}=x_{B e} c^{l} M_{B} ; \rho_{A e}^{v}$ and $\rho_{B e}^{v}$ denote mass densities of A and B in the gas phase, respectively, hence $\rho_{A e}^{v}=y_{A e} c^{v} M_{A}$ and $\rho_{B e}^{v}=y_{B e} c^{v} M_{B} ; f(t)$ denotes the position of the interface, and $v_{z}^{I}$ is the velocity of the gas-liquid interface. Thermodynamic equilibrium calculations at the interface (Prausnitz et al. [11]) or experimental data will be used to 
determine the interfacial compositions $y_{A e}$ and $x_{A e}$ as functions of $P$ and $T$. The molar densities in the gas and liquid phase can be calculated from standard equations of state (for example the Peng-Robinson equation of state; Peng and Robinson [12]). The problem is completed by specifying far-field conditions. At the place where gas in injected $(z=0)$ we have $y_{A}=0$, while initially and far from the interface in the liquid, the molar fraction is fixed $\left(x_{A}=x_{A \infty}\right)$. This results in the counter-diffusion of $\mathrm{A}$ and $\mathrm{B}$ in the liquid phase, in addition to their counter-diffusion in the gas phase.

The solution of this problem admits a similarity solution using the similarity variable $\eta=\frac{z}{2 \sqrt{\mathcal{D}_{A B}^{t}}}$. We also take

$$
f(t)=2 \lambda \sqrt{\mathcal{D}_{A B}^{v} t} \text { and } N_{z}^{v}(t)=\frac{\beta \sqrt{\mathcal{D}_{A B}^{v}}}{\sqrt{t}}
$$

where $\lambda$ is a dimensionless parameter to be determined, and $\beta$ has dimensions of molar density (mol/volume). In terms of these similarity variables, the mass balances become

$$
y_{A}^{\prime \prime}+2 y_{A}^{\prime}\left(\eta-\phi^{v}\right)=0 \text { in } 0<\eta<\lambda
$$

and

$$
x_{A}^{\prime \prime}+2 \alpha^{2} x_{A}^{\prime} \eta=0 \text { in } \lambda<\eta
$$

where primes denote derivative with respect to $\eta$ and we have denoted

$$
\phi^{v}=\frac{\beta}{c^{v}} \quad, \quad \alpha=\sqrt{\frac{\mathcal{D}_{A B}^{v}}{\mathcal{D}_{A B}^{l}}}
$$

The two equations (14) and (15) are subject to the interfacial conditions (10) and (11) (in the appropriate dimensionless form) and the following boundary conditions

$$
\begin{gathered}
y_{A}=0 \text { at } \eta=0 \quad \text { and } y_{A}=y_{A e} \text { at } \eta=\lambda \\
x_{A}=x_{A \infty} \text { at } \eta=\infty \quad \text { and } x_{A}=x_{A e} \text { at } \eta=\lambda
\end{gathered}
$$

Note that because the integration interval in the gas phase is $0<z<f(t)$ and $f(0)=0$, there is no need to satisfy an initial condition for that phase (in contrast to the problems 
considered by Bird et al. [6], Cussler [13], and Slattery and Mhetar [9]). The interfacial conditions (10) and (11) can be recast in the form

$$
\beta=-\lambda\left(\frac{\rho_{A e}^{l}-\rho_{A e}^{v}}{M_{A}}+\frac{\rho_{B e}^{l}-\rho_{B e}^{v}}{M_{B}}\right) \text { hence } \phi^{v}=-\lambda\left(\frac{1}{\epsilon}-1\right)
$$

where $\epsilon$ is the ratio of the molar densities, $\epsilon \equiv \frac{c^{v}}{c^{l}}$, and

$$
y_{A}^{\prime}-2 \phi^{v} y_{A e}+2 \lambda\left(y_{A e}-\frac{x_{A e}}{\epsilon}\right)=\frac{x_{A}^{\prime}}{\epsilon \alpha^{2}} \quad \text { at } \quad \eta=\lambda
$$

Away from the critical point, $\epsilon \ll 1$.

The solution of the above system is readily found to be

$$
\frac{y_{A}}{y_{A e}}=\frac{\operatorname{erf}\left(\eta-\phi^{v}\right)+\operatorname{erf}\left(\phi^{v}\right)}{\operatorname{erf}\left(\lambda-\phi^{v}\right)+\operatorname{erf}\left(\phi^{v}\right)} \quad \text { and } \quad \frac{x_{A}-x_{A \infty}}{x_{A e}-x_{A \infty}}=\frac{\operatorname{erf}(\alpha \eta)-1}{\operatorname{erf}(\alpha \lambda)-1}
$$

where the unknown parameter $\lambda$ satisfies the following transcendental equation

$$
\mu\left(y_{A e}-x_{A e}\right)+\frac{y_{A e} \exp \left[-\mu^{2}\right]}{\sqrt{\pi}[\operatorname{erf}(\mu)-\operatorname{erf}(\mu(1-\epsilon))]}=\frac{\left(x_{A \infty}-x_{A e}\right)}{\nu \sqrt{\pi}} \frac{\exp \left[-(\nu \mu)^{2}\right]}{\operatorname{erfc}(\nu \mu)}
$$

and where $\mu \equiv \frac{\lambda}{\epsilon}$ and $\nu \equiv \epsilon \alpha$, and erfc is the complementary error function. Equation (21) is the main result of this section. Its solution needs to be determined numerically. Before we proceed, however, it is useful to consider two special cases:

(i) In the limit $\nu \gg 1$, namely when $\alpha \gg \epsilon^{-1}, \mu \nu$ is at least of order 1 . Using asymptotic properties, equation (21) simplifies to

$$
\mu\left(x_{A \infty}-y_{A e}\right)=\frac{y_{A e} \exp \left[-\mu^{2}\right]}{\sqrt{\pi}[\operatorname{erf}(\mu)-\operatorname{erf}(\mu(1-\epsilon))]}
$$

We note that the solution of this equation is independent of the mole fraction in the liquid at the interface, $x_{A e}$, and in fact it coincides with the general equation (21) when $x_{A e}=x_{A \infty}$. Therefore, this limit can be viewed as either infinitesimally slow diffusion in the liquid phase $(\alpha \gg 1)$ or, equivalently, as a problem in which $\mathrm{B}$ does not dissolve in the liquid phase (in which case we need to take $x_{A \infty}=1$ for self-consistency). Thus, (22) also expresses the solution of the problem in the special case of negligible dissolution in the liquid. This can be further simplified by taking $\epsilon \ll 1$, as is typically the case, where a further expansion yields the result 


$$
\mu=\sqrt{\frac{y_{A e}}{2\left(x_{A \infty}-y_{A e}\right) \epsilon}} \text { hence } \quad \lambda=\sqrt{\frac{y_{A e} \epsilon}{2\left(x_{A \infty}-y_{A e}\right)}}
$$

From (23) we conclude that in the typical case, $\lambda$ is of order $\sqrt{\epsilon}$. Some further simplification leads to the dilute solution limit

$$
\lambda=\sqrt{\frac{y_{A e} c^{v} M_{A}}{2 \rho^{l}}}
$$

In this limit, the convective term vanishes and the concentration field is quasi-static, namely it satisfies a Laplace equation, the base-state profile for the mole fraction being linear. This result was used as the basis for a stability analysis presented in Tsimpanogiannis et al. [3].

(ii) In the other limit, $\nu \ll 1$, on the other hand, parameter $\mu$ is small. Using asymptotic properties we find from (21) the result

$$
\mu=\frac{y_{A e} \sqrt{\pi}}{2\left(x_{A \infty}-x_{A e}\right)} \alpha \quad \text { hence } \quad \lambda=\frac{y_{A e} \sqrt{\pi}}{2\left(x_{A \infty}-x_{A e}\right)} \alpha \epsilon
$$

This equation shows a linear dependence on $\alpha$, at small values of the latter. Given the definition of $\alpha^{2}$ as the ratio of vapor to liquid diffusivities, this case is not physically realistic. However, it serves to illustrate the fact that $\mu$ and $\lambda$ decrease (and vanish) as $\alpha$ decreases to zero (or as the mole fraction $x_{A e}$ decreases). Thus, accounting for diffusion in the liquid or for solubilization of the gas in the liquid will slow down the evaporation process, as originally conjectured. The specific extent of this decrease needs to be determined from the full equation (21), however.

\section{(ii). Spatially variable overall molar concentration}

In the general case, where we need to account for the dependence of the overall molar concentration $c^{l}$ on the mole fraction $x_{A}$, the previous system still admits a similarity solution in terms of the same similarity variable. Corresponding to (14) we now have a system of two equations

$$
-\eta \frac{d}{d \eta}\left(x_{A} c^{l}\right)+\frac{d N_{A z}^{l}}{d \eta}=0
$$




$$
-\eta \frac{d c^{l}}{d \eta}+\frac{d}{d \eta}\left(\frac{N_{A z}^{l}+\frac{c^{l}}{2 \alpha^{2}} \frac{d x_{A}}{d \eta}}{x_{A}}\right)=0
$$

where $c^{l}$ is a function of $x_{A}$ (as well as $T$, and $P$ ). These equations are to be solved subject to the boundary conditions (16) and (17) and the far-field condition

$$
N_{A}^{l}=0 \text { at } \eta=\infty
$$

Because the dependence $c^{l}\left(x_{A}\right)$ is generally non-trivial, an analytical solution of this twopoint boundary-value problem is not possible, in general. For its solution we used an iterative approach. First, the unknown value of $\lambda$ was guessed. Results from the previous section were used as initial guesses. Then, the boundary value problem (26)-(28) was solved by a shooting method, for which the solution at the far-field was also provided by the previous analytical solution. The numerical result was then inserted in the interface boundary conditions (10) and (11). If these were satisfied to the desired accuracy, the guessed value of $\lambda$ was accepted, otherwise, a new value was taken and the algorithm was iterated until a satisfactory convergence was reached. Numerical examples are shown below.

Although the general problem does not admit an analytical solution, it is of interest to note that analytical results are possible in the limit $\alpha \rightarrow \infty$. In this limit, it can be shown that the variation of $x_{A}$ occurs only in a boundary layer around $\eta=\lambda$ of thickness $\frac{1}{\alpha^{2}}$. In fact, the mole fraction varies in this boundary layer in a form that can be calculated exactly. After some manipulations, one can show that in this case, $x_{A}$ is implicitly given from the following

$$
(\eta-\lambda) \alpha^{2}=\frac{1}{2 \lambda c^{l}\left(x_{A \infty}\right)} \int_{x_{A C}}^{x_{A}} \frac{c^{l}(x) d x}{x_{A \infty}-x}
$$

Inserting the results from this equation in the interface conditions, one finds, after some algebra, that $\lambda$ satisfies the same equation as (22), except that now $\epsilon=\frac{c^{v}}{c^{l}\left(x_{A \infty}\right)}$. In other words, at large $\alpha$, the analytical solution for $\lambda$ previously developed under the assumption of a constant molar concentration holds, provided that the liquid molar concentration is evaluated at the far-field molar fraction $x_{A \infty}$. This result will be used below to bound the variation of $\lambda$ as a function of the thermodynamic variables. 


\section{RESULTS AND DISCUSSION}

Based on the previous results we explored the effects of the various parameters on the velocity of the interface. For numerical results, the following input data are needed: (i) the equilibrium molar fractions at the gas-liquid interface, for both liquid and gas phases, $\left(x_{A e}, y_{A e}\right)$; (ii) the molar concentrations of both liquid and gas mixtures, $\left(c^{l}, c^{v}\right)$, and their dependence as a function of composition, $T$ and $P$; and (iii) the ratio of the binary diffusion coefficient in the gas phase to the binary diffusion coefficient in the liquid phase, $\left(\frac{\mathcal{D}_{A B}^{v}}{\mathcal{D}_{A B}^{t}}\right)$.

At high pressures, we used experimentally reported data for the equilibrium molar fractions $x_{A e}$ and $y_{A e}$. These were obtained from Azarnoosh and McKetta [14].(n-decane/nitrogen); Poston and McKetta [15] (n-hexane/nitrogen); Sage et al. [16] (n-pentane/methane); and Besserer and Robinson [17] (n-pentane/carbon dioxide). At low pressures, $y_{A e}$ was approximated as a function of $T$ using the expression $y_{A e}=\frac{P_{A}^{s}(T)}{P}$, where $P_{A}^{s}(T)$ is the vapor pressure of the component A, obtained from published correlations (Prausnitz et al. [11]; Himmelblau [18]). For liquid and gas molar densities, high-pressure vapor-liquid equilibrium calculations were performed using the Peng-Robinson [12] cubic equation of state. However, since the equation of state was mainly used for volumetric calculations, a modified version, which includes a volume translation to improve volumetric predictions, was selected (Magoulas and Tassios [19]). For the case in which the liquid overall molar concentration was assumed spatially constant, its value corresponding to $x_{A e}$ was taken in the calculations. Reliable experimental data for the binary diffusion coefficients were obtained from Sigmund's correlation (Sigmund [20]; da Silva and Belery [21]). However we also considered the sensitivity of the results to various hypothetical cases in which $\alpha$ was kept constant.

First, we examined the validity of the assumption of spatially uniform liquid molar concentration. Figure 2 shows the variation of $\lambda$ with pressure at a fixed temperature, for the two different systems n-pentane/methane (Fig. 2a) and n-pentane/carbon dioxide (Fig. 2b) and for the two cases analyzed, one in which $c^{l}=c^{l}\left(x_{A e}\right)$ and another in which $c^{l}$ was allowed to vary with composition. Sigmund's correlation [20], [21] for the ratio of diffusivities was considered in both cases. As expected, the two solutions are close to each other at low pressures, but deviate at higher pressures. Their difference increases with pressure, but falls 
within the range of uncertainty of the diffusion coefficient variation, as will be shown below. Plotted in Figure 2 is also the theoretical result corresponding to $\alpha \gg 1$. As anticipated, this curve bounds from above the velocity of the interface, with the curve corresponding to the uniform concentration assumption serving as a lower bound. Overall, the trend in all cases is an increase of $\lambda$ as $P$ increases.

Subsequently, we examined the validity of the two asymptotic limits (23) and (25). Figure 3 is a plot of the parameter $\lambda$ vs. the square root of the diffusivity ratio, $\alpha$, for the system hexane-nitrogen at $310.93 \mathrm{~K}$ and 68.9 bar. Shown are results for both of the previous cases (assumption of spatially constant molar concentration and the more general case). The dotted lines represent the asymptotes described in the two equations (23) and (25), respectively, in the two limits of large and small $\alpha$, respectively. As expected, the asymptotic solutions represent quite well the behavior of the analytical solution as a function of the diffusivity ratio. The more general solution is also bounded at large $\alpha$ by the theoretical result (dash-dotted line), and is in general larger than the approximate analytical solution. At small values of $\alpha$, the general solution follows a linear variation with $\alpha$, similar to the analytical result (25). This dependence can, in fact, be proved analytically, by following a scaling analysis of the general problem in that limit. However, calculating the specific prefactor requires the numerical solution of the full problem. Similar behavior was found for other conditions or systems tested. Figure 3 reveals two important aspects: That, in general, $\lambda$ is of order $10^{-1}$ or less, as expected from (23); and that in its expected range of variation $\left(1,10^{2}\right)$, the diffusivity ratio has an effect on $\lambda$ which is of the order of two or three, but not of an order of magnitude. This observation essentially provides the answer to the question we raised in the introduction concerning the effect of solubilization and diffusion of component $B$ on the front velocity. In its absence, the rate of movement of the interface is faster. When it is taken into consideration, dissolution and diffusion will slow down the process. The amplification of this slowdown can be significant but it does not exceed an order of magnitude.

An increase in pressure will lead in general to an increase of the rate of movement of the interface. Figure 4 shows plots of $\lambda$ vs. pressure for the system n-pentane/methane at $310.93 \mathrm{~K}$, where the initial liquid is pure $\mathrm{n}$-pentane and where we used for simplicity the 
analytical solution. Plotted are predictions from (21) for various hypothetical cases: in some,

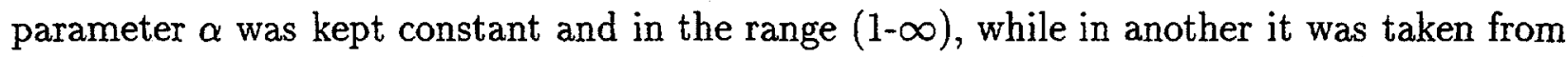
the correlation of Sigmund [20]. We note that the overall trend as the pressure increases is an increase in $\lambda$ and also an increase in its sensitivity to the diffusivity ratio. For the particular correlation of Sigmund [20], the maximum variation of parameter $\lambda$ is within a factor of two. Analogous results were found for the system n-pentane/carbon dioxide. For the latter, the sensitivity to the liquid diffusion is larger than for the n-pentane/methane shown in Figure 4 in the same range of pressure. For example, for the pressure of 63 bar, where experiments were reported by Le Romancer et al. [5], the effect of diffusion was found to slow down the evaporation front by a larger extent than in the case of n-pentane/methane. This result is consistent with the experimental findings.

The effect of the initial mole fraction of the liquid phase on the evaporation rate of the previous mixture at $P=103.4$ bar is shown in Figure 5, where again the simpler analytical solution was used. As expected, the highest rate corresponds to an initial liquid mixture at equilibrium $\left(x_{A \infty}=x_{A e}\right)$, since in this case there is no dissolution of $\mathrm{B}$ in the liquid. Increasing $x_{A \infty}$ leads to an increased dissolution and to an accompanying decrease in the evaporation rate. Consistent with the previous plots, the decrease is generally within a factor of two of the value at equilibrium, regardless of the value of the diffusivity ratio.

More important is the effect of temperature, shown in Figures 6 and 7. Figure 6 shows high-pressure results for the two pairs, $n$-hexane/nitrogen and $n$-decane/nitrogen, over a range of temperatures, for various assumptions regarding $\alpha$, and where the analytical solution is used. We note that the sensitivity to temperature is larger than that of either the pressure or of the initial mole fraction discussed above. This trend is expected. Results in Figure 7 correspond to low pressures $(P=1 \mathrm{bar}$ ), for the pairs n-pentane/nitrogen and $\mathrm{n}$-decane/nitrogen. The effect of temperature on $\lambda$ is quite significant. In fact, as the system approaches its boiling point, $\lambda$ becomes infinitely large (although, strictly speaking, the present analysis will break down near that point). Under these conditions, the dependence of $\lambda$ on temperature can be extracted by using (21) in the limit of large $\mu$. Then, it is not difficult to show the result 


$$
\lambda=\epsilon \sqrt{\frac{\ln \left(\frac{1-\epsilon}{1-y_{A e}}\right)}{2 \epsilon-\epsilon^{2}}}
$$

where we have assumed $x_{A \infty}=1$. This equation describes the asymptotic behavior of $\lambda$ as the temperature approaches the boiling point (where $y_{A e}=1$ ). The latter equation appears to describe very well the behavior of $\lambda$ in this region (see Figure 7).

\section{CONCLUSIONS}

In this paper we presented a simple 1-D model of the evaporation of a liquid, immobilized or trapped in a porous medium, into a flowing gas outside the medium. The solution of this problem is useful as a building block for the treatment of the more general problem, where pore microstructure, and capillary and viscous effects are taken into account (see [3] for an application in the drying context). We have focused in particular on the effect of dissolution of gas B and its counter-diffusion in liquid A. It was found that the effect on evaporation is to slow down the evaporation rate, as also noted in related experiments in porous media. However, in the typical applications the degree of this reduction is generally within a factor of two or three, but not of an order of magnitude.

\section{ACKNOWLEDGMENTS}

The research of INT and YCY was supported by DOE Contract No. DE-AC2699BC1521. The research of AKS was partially funded by the JOULE Programme of the European Commission (Contract JOF3-CT95-0008). All these sources of support are gratefully acknowledged. 


\section{References}

[1] Le Gallo, Y.; Le Romancer, J.F.; Bourbiaux, B.; Fernandes, G. Mass Transfer in Fractured Reservoirs During Gas Injection: Experimental and Numerical Modeling. SPE Annual Technical Conference and Exhibition, San Antonio, TX, October 1997; paper SPE 38924.

[2] Ho, C.K.; Lin, S.-W.; Udell, K.S. Propagation of Evaporation and Condensation Fronts During Multicomponent Soil Vapor Extraction. J. Contam. Hydrol. 1994, 16, 381.

[3] Tsimpanogiannis, I.N.; Yortsos, Y.C.; Poulou, S.; Kanellopoulos, N.; Stubos, A.K. Scaling Theory of Drying in Porous Media. Phys. Rev. E. 1999, 59, 4353.

[4] Shaw, T.M. Drying as an Immiscible Displacement Process with Fluid Counterflow. Phys. Rev. Lett. 1987, 59, 1671.

[5] Le Romancer, J.F.; Defives, D.; Kalaydjian, F.; Fernandes, G. Influence of the Diffusing Gas on the Mechanism of Oil Recovery by Gas Diffusion in Fractured Reservoir.IEA Collaborative Project of Enhanced Oil Recovery Workshop and Symposium, Bergen, Norway, 28-31 August 1994.

[6] Bird, R.B.; Stewart, W.E.; Lightfoot, E.L. Transport Phenomena; Wiley: New York, 1960.

[7] Slattery, J.C. Momentum, Energy and Mass Transfer in Continua; Krieger: New York, 1978. 
[8] Taylor, R.; Krishna, R. Multicomponent Mass Transfer; Wiley: New York, 1993.

[9] Slattery, J.C.; Mhetar, V.R. Unsteady-State Evaporation and the Measurement of a Binary Diffusion Coefficient. Chem. Eng. Sci. 1997, 52, 1511.

[10] Mhetar, V.R.; Slattery, J.C. The Stefan Problem of a Binary Liquid Mixture. Chem. Eng. Sci. 1997, 52, 1237.

[11] Prausnitz, J.M.; Lichtenhaler, K.N.; de Azevedo, E.G. Molecular Thermodynamics of Fluid-Phase Equilibria; Prentice-Hall: Englewood Cliffs, 1986.

[12] Peng, D.Y; Robinson, D.R. A New Two-Constant Equation of State. Ind. Eng. Chem. Fundamentals. 1976, 15, 59.

[13] Cussler, E.L. Diffusion: Mass Transfer in Fluid Systems; $2^{\text {nd }}$ ed.; Cambridge University Press: Cambridge, 1997.

[14] Azarnoosh, A.; McKetta, J.J. Nitrogen-n-Decane System in the Two-Phase Region. Journal Chem. Eng. Data. 1963, 8, 494.

[15] Poston, R.S; McKetta, J.J. Vapor-Liquid Equilibrium in the n-Hexane- Nitrogen System. Journal Chem. Eng. Data. 1966, 11, 364.

[16] Sage, B.H.; Reamer, H.H.; Olds, R.H.; Lacey, W.N. Volumetric and Phase Behavior of Methane-n-Pentane System. Ind. Eng. Chem. 1942, 34, 1108. 
[17] Besserer, G.J.; Robinson, D.B. Equilibrium-Phase Properties of the n-Pentane/Carbon Dioxide System. Journal Chem. Eng. Data. 1973, 18, 416.

[18] Himmelblau, D.M. Basic Principles and Calculations in Chemical Engineering; Prentice Hall: New Jersey, 1996.

[19] Magoulas, K; Tassios, D. Thermophysical Properties of n-Alkanes from $C_{1}$ to $C_{20}$ and their Predictions for Higher Ones. Fluid Phase Equilibria. 1990, 56, 119.

[20] Sigmund, P.M. Prediction of Molecular Diffusion at Reservoir Conditions. Part I. Measurement and Prediction of Binary Dense Gas Diffusion Coefficients. J. Can. Pet. Tech. April-June 1976, 48.

[21] da Silva, F.V.; Belery, P. Molecular Diffusion in Naturally Fractured Reservoirs: A Decisive Recovery Mechanism. SPE Annual Technical Conference and Exhibition, San Antonio, TX, October 1989; paper SPE 19672. 


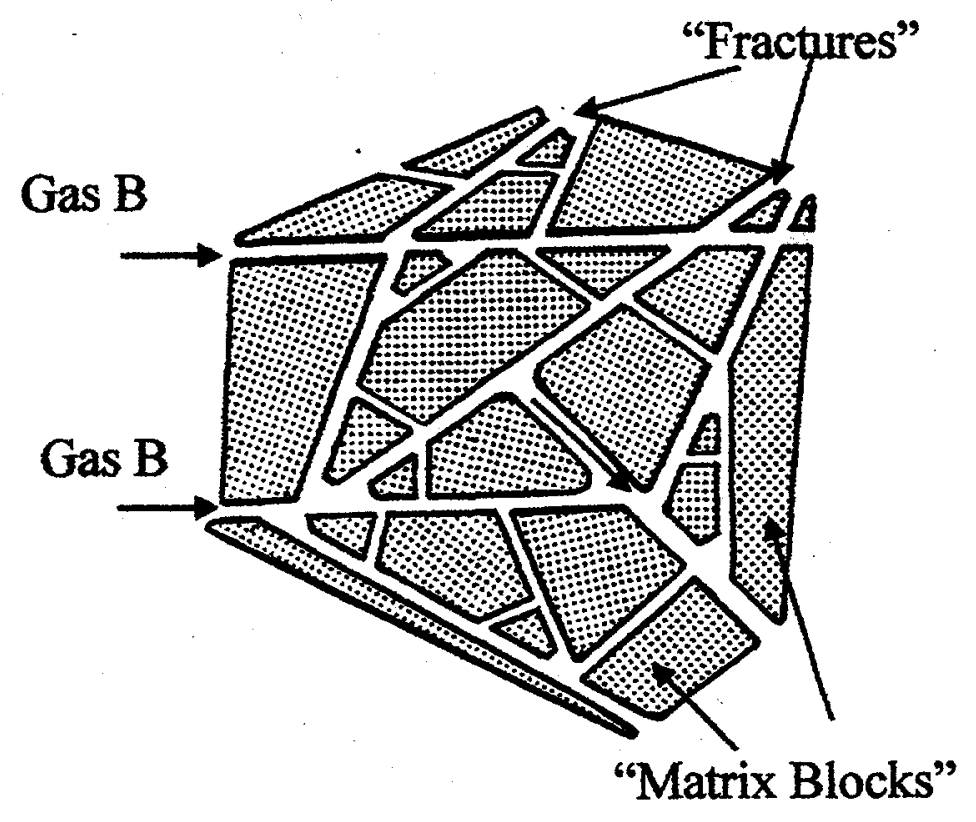

(a)

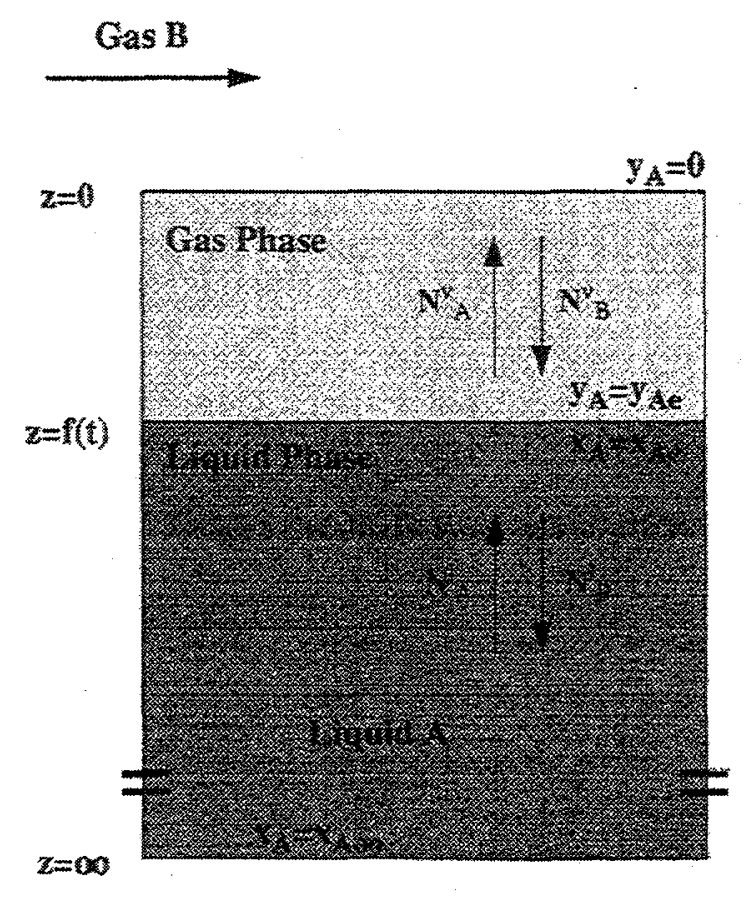

(b)

Figure 1: Schematic of the configuration considered. (a) Gas B flowing in a high permeability fracture network causes evaporation and diffusion of liquid A, trapped in matrix blocks (b) One-Dimension approximation, in which liquid $\mathrm{A}$ evaporates and diffuses in the gas phase, containing component B. The latter dissolves and diffuses in the liquid phase. 

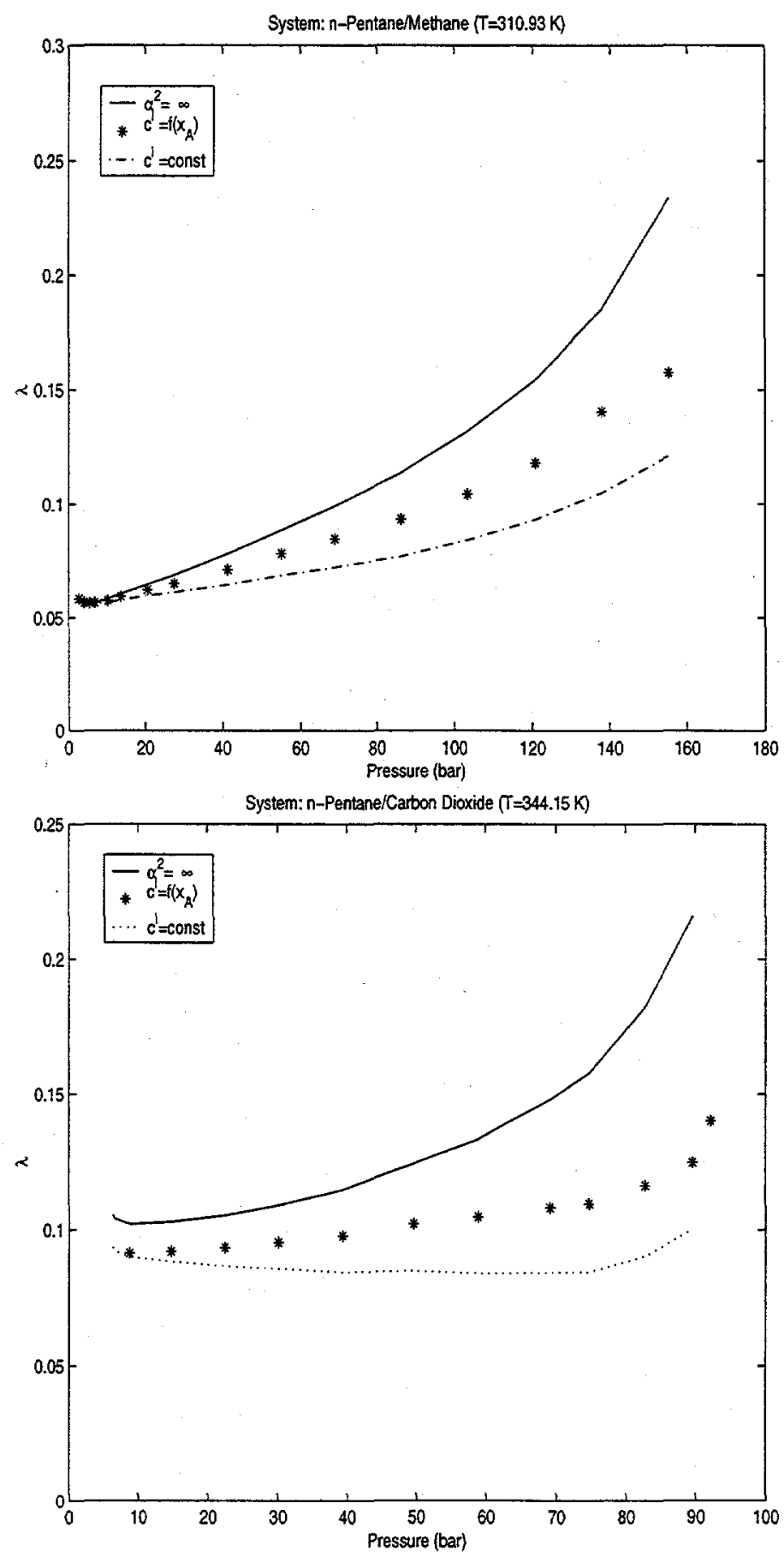

Figure 2: The effect of pressure on the velocity of the evaporation front for the systems: (a) n-pentane/methane at $310.93 \mathrm{~K}$, and (b) n-pentane/carbon dioxide at $344.15 \mathrm{~K}$. The dashed-dotted line corresponds to the analytical solution where $c^{l}=c^{l}\left(x_{A e}\right)$, the solid line is the theoretical result at large $\alpha$, while ${ }^{*}$ denotes the numerical results for the general case where $c^{l}=c^{l}\left(x_{A}\right)$. 


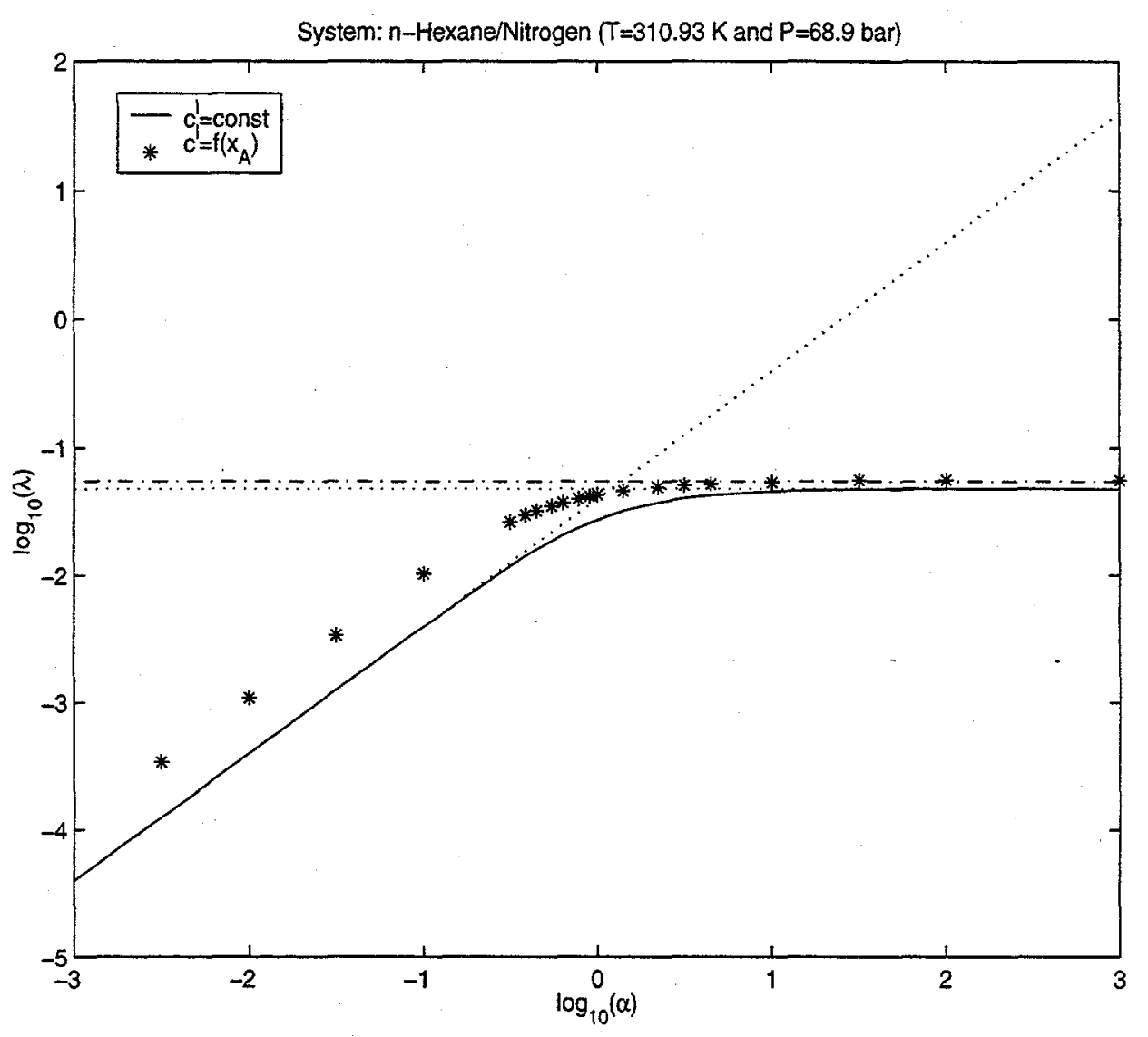

Figure 3: The effect of the ratio of diffusivities $\alpha^{2}=\frac{\mathcal{D}_{A B}^{v}}{\mathcal{D}_{A B}^{t}}$ (gas-to-liquid), on the velocity of the evaporation front for the system $\mathrm{n}$-hexane/nitrogen at $310.93 \mathrm{~K}$ and 68.9 bar. The dotted lines are the asymptotes expressed by (23) and (25), respectively, the dash-dotted line is the theoretical prediction for the general problem at large $\alpha$. 


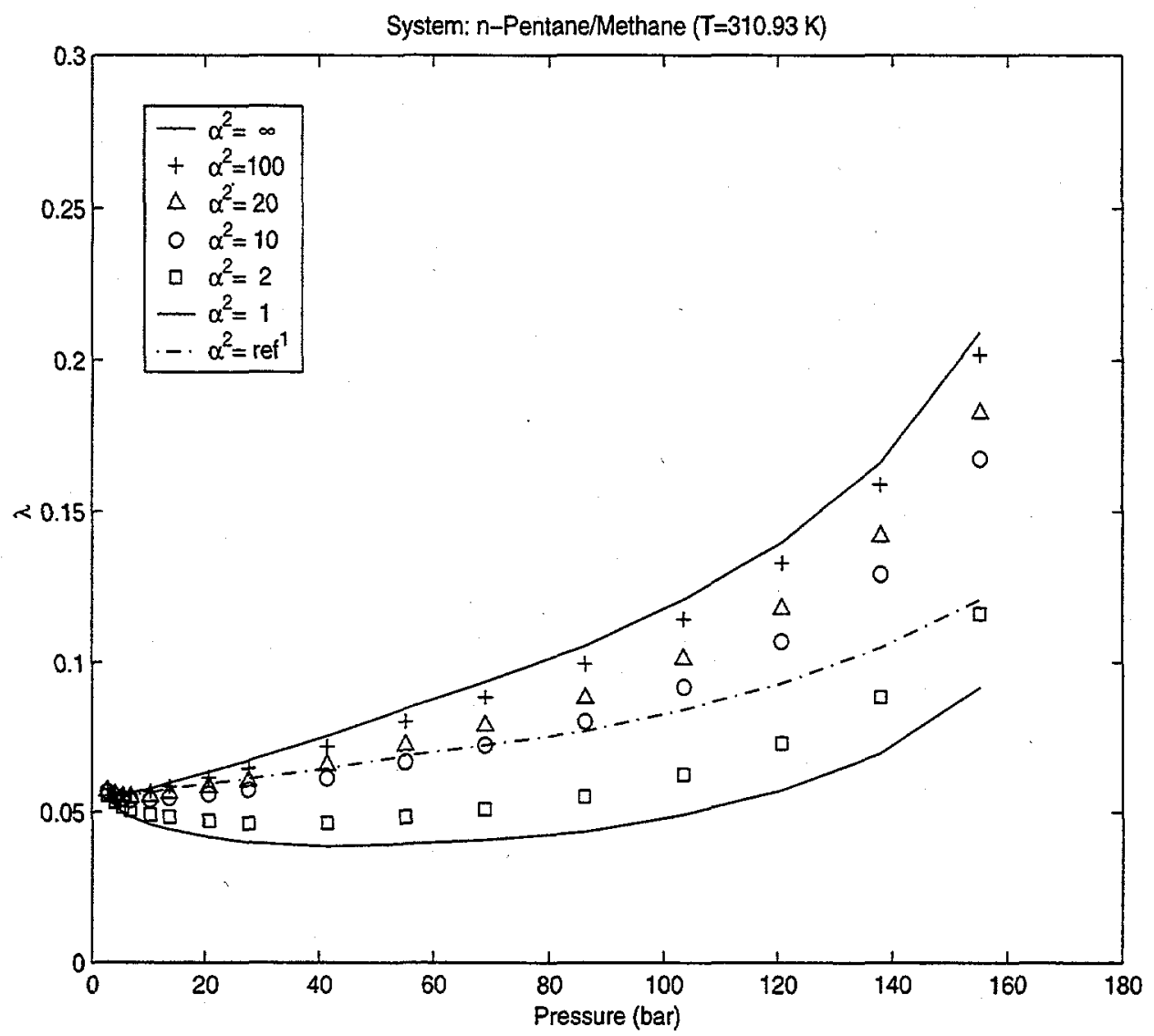

Figure 4: The effect of pressure on the velocity of the evaporation front for the system $\mathrm{n}$-pentane/methane at $310.93 \mathrm{~K}$. The two solid lines correspond to simulations where the ratio of diffusivities is equal to $\infty$ and 1, respectively. These curves envelope various curves corresponding to ratio of diffusivities equal to $100,20,10$ and 2 (denoted with symbols,$+ \triangle$, $\circ$, and $\square$ respectively), as well as a case ( $\mathrm{ref}^{1}$ ) using the correlation of Sigmund [20] (denoted by dashed-dotted line). 


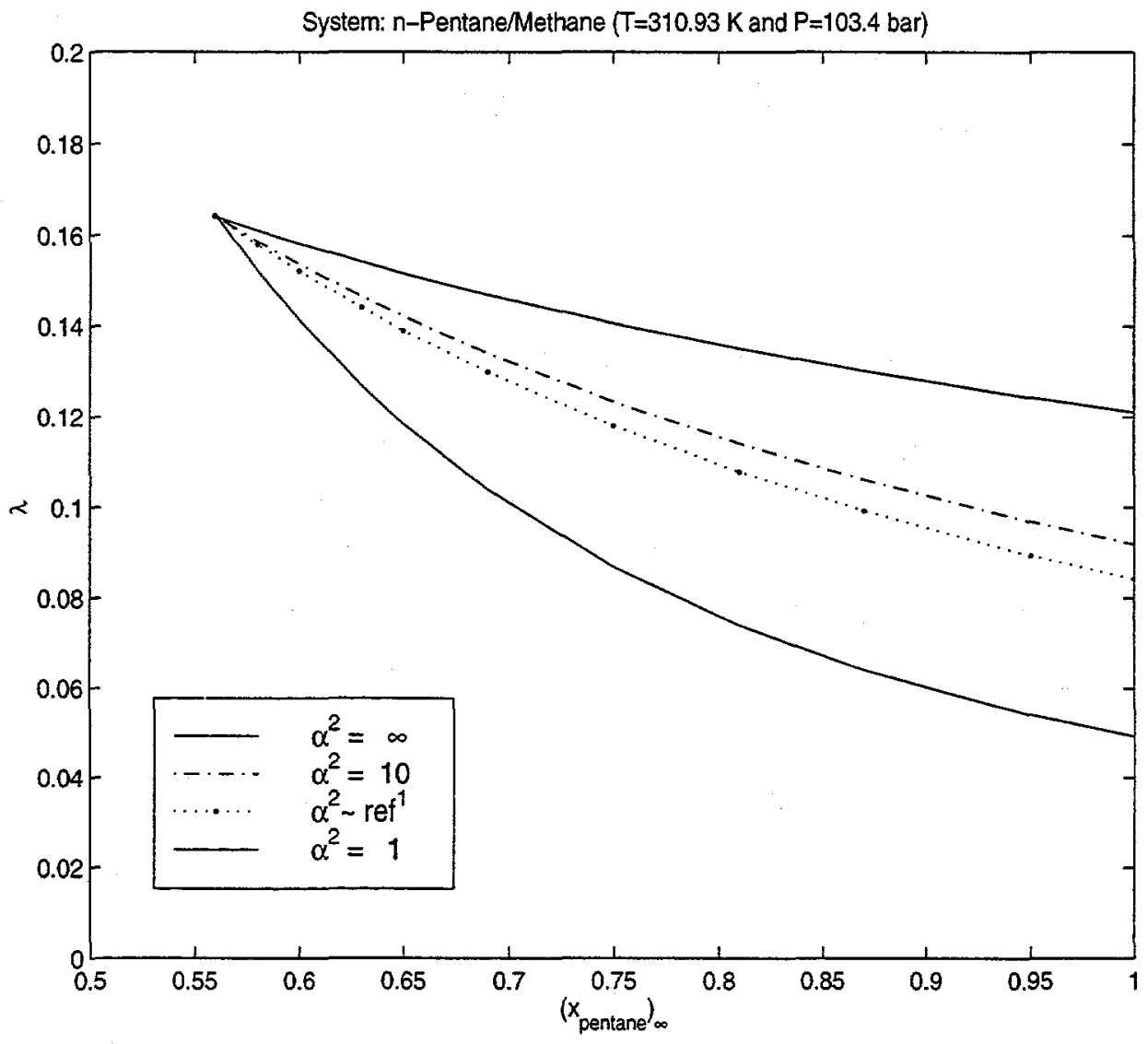

Figure 5: The effect of initial liquid composition on the velocity of the evaporation front for the system n-pentane/methane at $310.93 \mathrm{~K}$ and $103.4 \mathrm{bar}$. The various curves shown correspond to the values of the ratio of diffusivities equal to $\infty, 10$, and 1 , as well as a case $\left(\mathrm{ref}^{1}\right)$ using the correlation of Sigmund [20] (denoted by solid, dash-dotted, solid and dotted, respectively). 


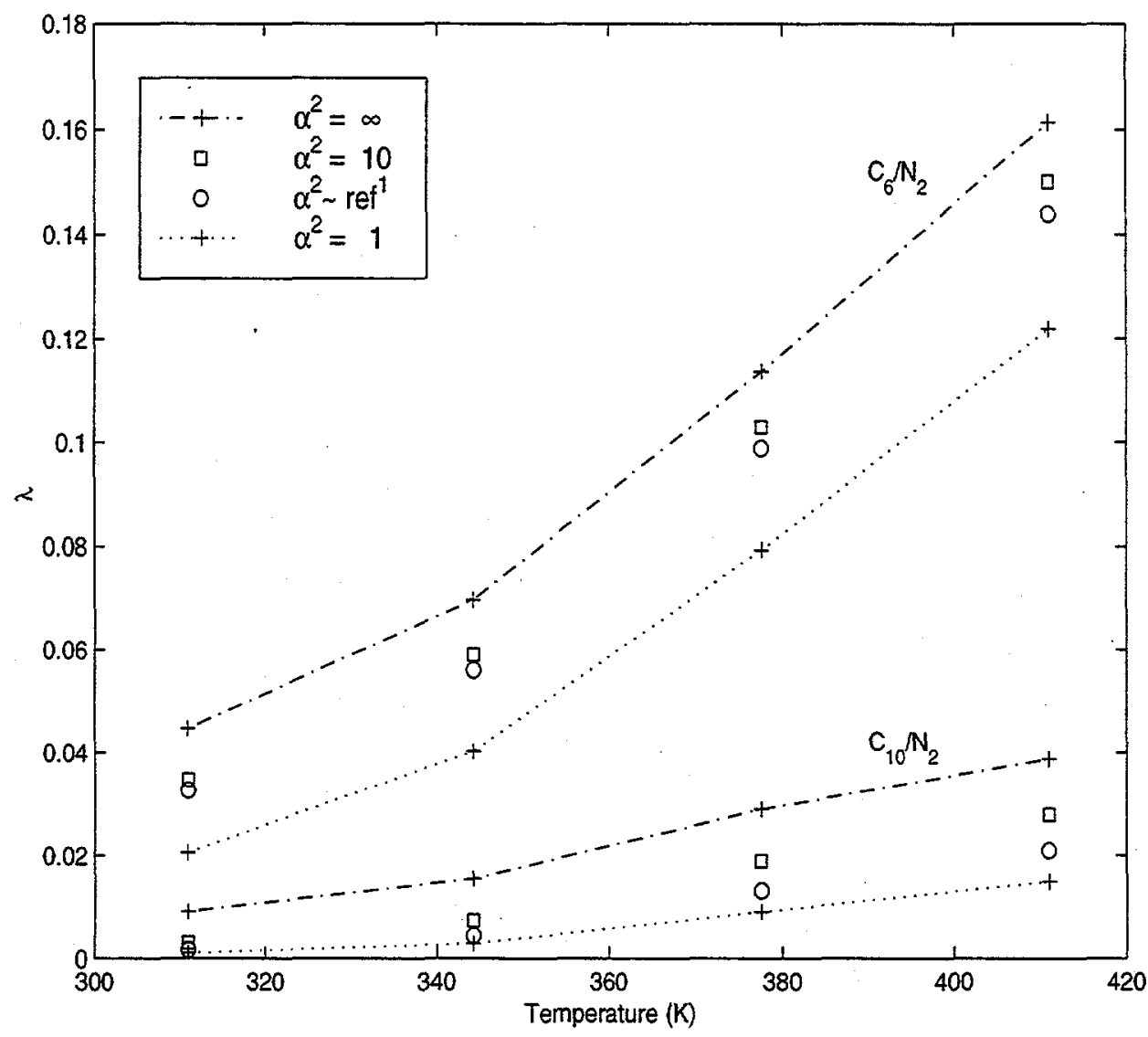

Figure 6: The effect of temperature on the velocity of the evaporation front for the two different systems n-hexane/nitrogen and n-decane/nitrogen at 103.4 bar. The various curves shown correspond to the values of the ratio of diffusivities equal to $\infty, 10$, and 1 , as well as a case $\left(\operatorname{ref}^{1}\right)$ using the correlation of Sigmund [20] (denoted by $-\cdot+, \square, \cdots+$, and $\circ$, respectively). 


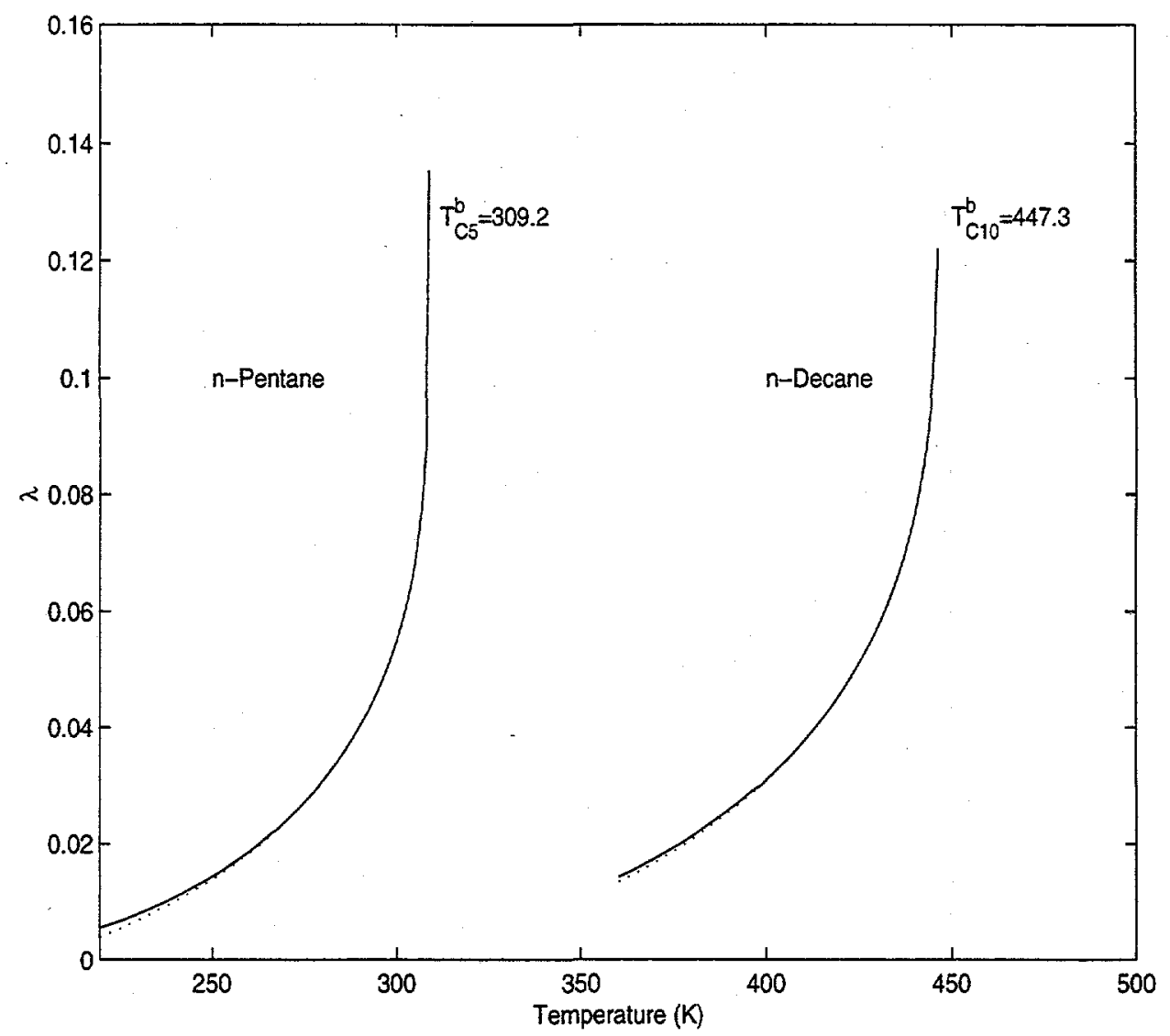

Figure 7: The effect of temperature on the velocity of the evaporation front for the systems $\mathrm{n}$-pentane/nitrogen and n-decane/nitrogen at $\mathrm{P}=1$ bar. The dotted line corresponds to the approximation (30). 